\title{
On optimal stress for shaft-hub connections (polygon connections)
}

\section{Pedersen, Niels Leergaard}

Published in:

Journal of Strain Analysis for Engineering Design

Link to article, DOI:

$10.1177 / 0309324720969530$

Publication date:

2021

Document Version

Peer reviewed version

Link back to DTU Orbit

Citation (APA):

Pedersen, N. L. (2021). On optimal stress for shaft-hub connections (polygon connections). Journal of Strain Analysis for Engineering Design, 56(4), 195-205. https://doi.org/10.1177/0309324720969530

\section{General rights}

Copyright and moral rights for the publications made accessible in the public portal are retained by the authors and/or other copyright owners and it is a condition of accessing publications that users recognise and abide by the legal requirements associated with these rights.

- Users may download and print one copy of any publication from the public portal for the purpose of private study or research.

- You may not further distribute the material or use it for any profit-making activity or commercial gain

- You may freely distribute the URL identifying the publication in the public portal

If you believe that this document breaches copyright please contact us providing details, and we will remove access to the work immediately and investigate your claim 


\title{
On optimal stress for shaft-hub connections (polygon connections)
}

\author{
Niels Leergaard Pedersen \\ Dept. of Mechanical Engineering, Solid Mechanics \\ Technical University of Denmark \\ Nils Koppels Allé, Building 404, DK-2800 Kgs. Lyngby, Denmark \\ email: nlp@mek.dtu.dk
}

\begin{abstract}
In machine elements many different methods for connecting shaft and hub are specified in different standards. A not so common shaft hub connection is the polygon profile as specified in DIN 32711 (P3G) and DIN 32712 (P4C). This type of connection is an alternative to key or spline connections. The practical point of the connection is the easy assembly and disassembly, but as for all shaft hub connections the drawback is the high stress leading to a limited strength of these connections. The polygon connection design is to some extent due to an old production method, i.e. it is not design/optimized for reducing the maximum stress. In the present paper it is shown how the connection strength can be improved significantly by the use of shape optimization. The focus here is both on the highest contact stress (compression) but also on the largest tensile stress. Overall reduction in the stress in the order of $20 \%$ to $40 \%$ is found relative to standard design.
\end{abstract}

Key words: Machine elements, polygon connection, torsion, stress concentration, FEA, 2D/3D.

\section{Introduction}

Many different designs and methods exist for shaft hub connections, all with the purpose of transmitting power. Basically they all fall into two different categories, either transmission by frictional force, e.g. as an interference or cone fit, or alternatively the shaft and hub are geometrically locked. The latter is also termed a positive connection. The typical design of positive connections use keys or the keys are built into the shaft, i.e. a spline connection. The designs used by the industry are defined in different standards. The geometric shape used in the standards are typically straight lines and circular arches although some splines are designed with the involute shape used typically in gear design. The typical design assumption is that for the bearing stress, which is compressive, we do not need to consider fatigue.

Although the bearing stress is predominating compressive there will be, depending on the design, areas with tensional stress or shear stress that can lead to fatigue failure of the hub or shaft. It can therefore be argued that for the positive connections between shaft and hub the strength (fatigue) is to a large extent controlled by the stress concentrations and the stress singularities in the contact. The maximum stress is controlled by two factors: firstly, the shape at the point of maximum stress that defines the stress concentration factor which is a local quantity, and secondly, the amount of load that is transferred to the point with the stress concentration. 
The last point is dictated by the more global design, i.e. the placement of the boundary condition and the external load and how the load is transferred between these two. This aspect of two factors controlling the stress concentration size is also discussed in (Pedersen 2019).

As described in (Filemon 1959) polygon profiles date back to the development of a production machine in 1939. The reason for the specific shape is therefore primarily related to the possibility for production and not the possibility for minimizing stress. In the resent years there have been an increased interest in the polygon profiles as also seen in the literature, see e.g. based on experimental work (Fessler \& Moore 1996) or finite element analysis in (Czyzewski \& Odman 1988), (Kahn-Jetter, Hundertmark \& Wright 2000) and (Großmann \& Ziaei 2004). Presently, full 3D analysis has also been performed using the boundary element method (?) and (Citarella \& Perrella 2015), and using the finite element method (Bhatta \& Reffeor 2017). The complexity in the different papers varies, i.e. is tolerance in the connection included or not? Verification and comparison to experimental work is also presented. For all of the referenced papers no optimization has been performed, i.e. the shape is fixed. However, the eccentricity has been varied, see (Großmann 2007). The use of other profiles can be found in e.g. (Ziaei 2010), and to some extent the subject of the present paper is also related to the design of screw heads. For screw heads the design criterion is not fatigue, this is only relevant for the screw head drivers.

Design optimization of machine elements is an important subject with potentially large impact on the strength. The geometry is typically defined in standards to ensure that the different elements can function together. The purpose of the standards is also to ensure that the element strength is high. The variation in the used shape parameterization is not large and is to some extent controlled by previous production possibilities. Although we at the present, can produce complicated geometries directly from CAD/CAM this possibility has not had a large impact on the standard design of machine elements. For new design suggestions it is important that the design is practical, e.g. not defined by highly complicated parameterizations and similarities in the design for overall size changes are preferred. Examples of optimization of standard machine elements can be found in e.g. (Pedersen 2016) or (Pedersen 2013).

In the present paper we apply the finite element method (FEM) to evaluate the strength of the design. The optimized designs are compared to the design defined in the standard. The overall objective is to reduce the stress concentration. For the specific problem of shaft-hub connections, the contact geometry is also the one that we want to design. It is generally so that we can state that a given shape is optimized if the stress along the shape is constant, assuming that the specific shape is where we have the highest stress that controls the strength. The stress evaluation is important and it is essential that a FE model mesh refinement result in a constant maximum stress, so that the stress level is verified. For this reason the shape parameterization should be disconnected from the FE model nodes so that the nodes in a refined model is placed correctly on the defined shape. This important point is also described in (Ding 1986). One benefit from this aspect is that a parameterization with a limited number of parameters can be used. This also seen in relation to the practicality of new design proposals for machine elements. As done in previous papers the super elliptical parameterization is used, and here used in a variation relative to what we have previously applied. See e.g. (Pedersen 2010) and (Pedersen 2011) also in relation to optimization of shaft-hub connection. In these papers the modelling was without contact modelling. The results presented in the present paper show that large improvements, in the order of $20 \%$ to $40 \%$, can be found by shape optimization.

The paper is organized as follows. In Section 2 the mathematical definition of the polygon shape is defined and the limits to the design is discussed in relation to (DIN 32711 1979) and (DIN 32712 1979). The numerical evaluation is presented in Section 3. The difference between a $2 \mathrm{D}$ and a full 3D contact modelling is discussed in relation to the design optimization 
presented in Section 4. The conclusions are given in Section 5.

\section{Mathematical definition}

The polygon profile is defined by the shape in 2D, examples are shown in Figure 1, and the mathematical definition can in parametric form be given by

$$
\begin{array}{r}
\left\{\begin{array}{l}
x \\
y
\end{array}\right\}=\left(\frac{D_{m}}{2}-e \cos (n t)\right)\left\{\begin{array}{c}
\cos (t) \\
\sin (t)
\end{array}\right\}+ \\
n e \sin (n t)\left\{\begin{array}{c}
-\sin (t) \\
\cos (t)
\end{array}\right\}, t \in[0: 2 \pi]
\end{array}
$$

where $n$ is the polygon side number, i.e. $n=3$ for the trilobe, $D_{m}$ is the mean diameter and $e$ is the eccentricity defined as

$$
e=\frac{D_{o}-D_{i}}{4}
$$

here $D_{o}$ and $D_{i}$ are the outer and inner diameter respectively, i.e.

$$
D_{o}=D_{m}+2 e \quad D_{i}=D_{m}-2 e
$$

Examples of a trilobe, $n=3(\mathrm{P} 3 \mathrm{G})$, and a profile for $n=4$ is shown in Figure 1 .

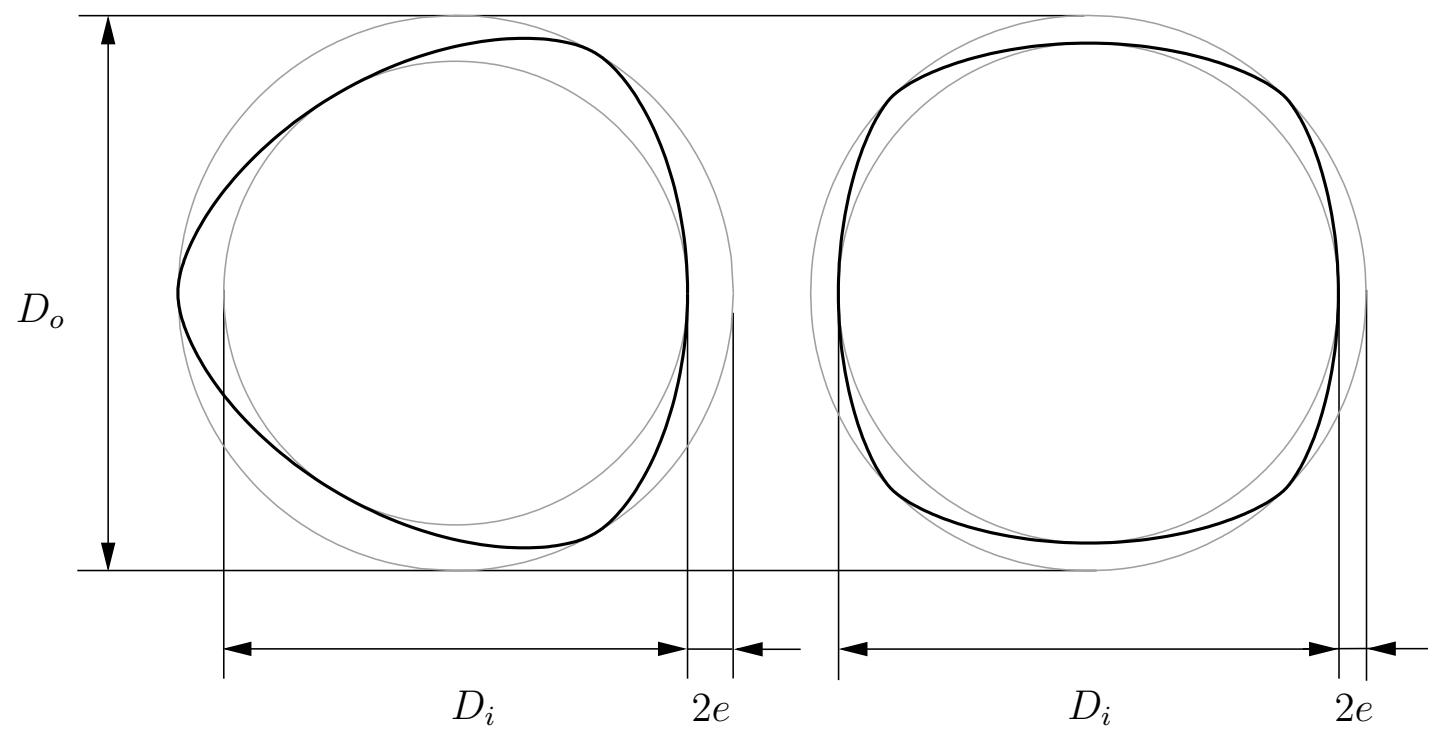

Figure 1: Left a P3G $(n=3)$ profile right a profile with $n=4$.

Alternatively, the profile can be given in polar form as

$$
\begin{aligned}
r(t) & =\sqrt{\left(\frac{D_{m}}{2}-e \cos (n t)\right)^{2}+(n e \sin (n t))^{2}} \\
\theta(t) & =t+\alpha \\
\alpha & =\arctan \left(\frac{n e \sin (n t)}{D_{m} / 2-e \cos (n t)}\right)
\end{aligned}
$$


The definition presented in (1) does not guarantee a closed curve that does not overlap. The boundaries on the eccentricity, that ensures a curve that does not overlap, can be found if the curve curvature becomes zero defined by

$$
\frac{d x}{d t} \frac{d^{2} y}{d t^{2}}-\frac{d y}{d t} \frac{d^{2} x}{d t^{2}}=0 \quad \Rightarrow \quad \frac{D_{m}}{2}+e\left(n^{2}-1\right) \cos (n t)=0
$$

which leads to the following boundaries on the eccentricity (assuming positive eccentricity values)

$$
0 \leq e \leq \frac{D_{m}}{2\left(n^{2}-1\right)}
$$

In the standard DIN 32712 a profile (P4C) is defined, this profile does not follow (1) entirely, higher eccentricity values are selected and at the same time a maximum diameter for the profile is selected, this results in profiles with 8 points with discontinuity in the slope.

The connection purpose is the transmission of torque. For a positive connection the normal force primarily transmits the torque. An important parameter is therefore the angle between the normal vector and the position vector (from centre of shaft to point on surface). This angle is equal to the previously defined, $\alpha$, and is termed the pressure angle since it defines the torque to force ratio. The maximum value for $\alpha$ is found for

$$
t=\arccos \left(\frac{2 e}{D_{m}}\right)
$$

leading to

$$
\tan \left(\alpha_{\max }\right)=\left(\frac{2 n e}{\sqrt{\left.D_{m}^{2}-4 e^{2}\right)}}\right)
$$

Selecting the maximum value in (8) gives

$$
\begin{aligned}
& n=3 \Rightarrow \alpha_{\max }=20.70^{\circ} \\
& n=4 \Rightarrow \alpha_{\max }=14.96^{\circ}
\end{aligned}
$$

These values can be compared to e.g. a straight sided spline where $\alpha=90^{\circ}$ and an interference fit where $\alpha=0^{\circ}$. The lower value for $n=4$ is probably why the standard P4C profile is modified to use higher numbers for the eccentricity leading to a higher pressure angle. However, the discontinuity in the slope leads to high stress concentrations, in theory of infinite magnitude.

With tolerances, here defined by different values of mean diameter and eccentricity for hub and shaft (given by $e_{h}, D_{h}, e_{s}$ and $D_{s}$ ), there will be and initial (rigid body) relative rotation between shaft and hub before contact. The condition for the point of contact is that the two profiles have the same radius, defined by (4), i.e.

$$
\begin{gathered}
\left(\frac{D_{s}}{2}-e_{s} \cos \left(n t_{s}\right)\right)^{2}+\left(n e_{s} \sin \left(n t_{s}\right)\right)^{2}= \\
\left(\frac{D_{h}}{2}-e_{h} \cos \left(n t_{h}\right)\right)^{2}+\left(n e_{h} \sin \left(n t_{h}\right)\right)^{2}
\end{gathered}
$$


The second condition for calculating the values of $t_{s}$ and $t_{h}$ defining the point of contact is that the pressure angle (or curve slope) is identical, i.e.

$$
\alpha_{s}=\alpha_{h}
$$

which from (6) gives

$$
\frac{n e_{s} \sin \left(n t_{s}\right)}{D_{s} / 2-e_{s} \cos \left(n t_{s}\right)}=\frac{n e_{h} \sin \left(n t_{h}\right)}{D_{h} / 2-e_{h} \cos \left(n t_{h}\right)}
$$

The solution to (11) and (13) is that

$$
\begin{aligned}
n e_{s} \sin \left(n t_{s}\right) & =n e_{h} \sin \left(n t_{h}\right) \\
D_{s} / 2-e_{s} \cos \left(n t_{s}\right) & =D_{h} / 2-e_{h} \cos \left(n t_{h}\right)
\end{aligned}
$$

These conditions for contact could alternatively also be realised directly from (1), as seen the points on the curve are defined by the scaled sum of two perpendicular unit vectors and the tangent to the curve is given by

$$
\begin{aligned}
\left\{\begin{array}{l}
d x / d t \\
d y / d t
\end{array}\right\}= & \left(\frac{D_{m}}{2}+e\left(n^{2}-1\right) \cos (n t)\right) . \\
& \left\{\begin{array}{c}
-\sin (t) \\
\cos (t)
\end{array}\right\}, t \in[0: 2 \pi]
\end{aligned}
$$

i.e. parallel to the last unit vector in (1), the only possibility to fulfil that the curves meet at a point and have the same tangent is therefore that the multiplication factors to the unit vectors are identical for the two curves leading to the conditions (14) and (15). By the definition of relative numbers (as also done in (Czyzewski \& Odman 1988))

$$
\begin{aligned}
& \delta=\frac{D_{h}-D_{s}}{e_{s}} \\
& \psi=\frac{e_{h}}{e_{s}}
\end{aligned}
$$

We find by the solution of (14) and (15)

$$
\begin{aligned}
& \cos \left(n t_{s}\right)=\frac{\psi^{2}-1-\delta^{2} / 4}{\delta} \\
& \cos \left(n t_{h}\right)=\frac{\psi^{2}-1+\delta^{2} / 4}{\delta \psi}
\end{aligned}
$$

and the relative rotation angle, $\Delta \theta$, is directly given, using (5), by

$$
\begin{array}{r}
\Delta \theta=t_{s}-t_{h}=\frac{1}{n}\left(\arccos \left(\frac{\psi^{2}-1-\delta^{2} / 4}{\delta}\right)-\right. \\
\left.\arccos \left(\frac{\psi^{2}-1+\delta^{2} / 4}{\delta \psi}\right)\right)
\end{array}
$$


The equation is only valid for a range of relative values given by that outer and inner hub diameter must be greater than the outer and inner shaft diameter respectively and the outer shaft diameter must be greater than the inner hub diameter leading to

$$
|1-\psi|<\delta<1+\psi
$$

\section{Numerical evaluation of maximum stress}

The stress evaluation is in the present paper performed numerically using the finite element method (FEM) and the numerical tool COMSOL (COMSOL AB 1986 -). The contact is modelled using the penalty method and the convergence of the contact modelling is performed by COMSOL. The material data used for all analysis is

$$
E=2.1 \cdot 10^{11} \mathrm{~N} / \mathrm{m}^{2}, \quad \nu=0.3
$$

where $E$ is modulus of elasticity and $\nu$ is Poisson's ratio.

The geometric data and load used for the first example is

$$
\begin{array}{lll}
D_{m}=0.1 \mathrm{~m}, & e=0.045 D_{m}, & D_{h o}=2 D_{m} \\
W_{h}=D_{m}, & T=200 \mathrm{Nm} &
\end{array}
$$

where $D_{h o}$ is the outer hub diameter, $W_{h}$ is the hub width and $T$ is the applied torque. The value of $D_{m}$ and the torque is applied to all numerical examples. The stress is shown as a stress concentration factor defined as

$$
K_{v M}=\frac{\sigma_{\max }^{v M}}{\sigma_{\text {nom }}^{v M}}
$$

where $\sigma_{\max }^{v M}$ is the maximum von Mises stress and the nominal von Mises stress is defined relative to a circular shaft, i.e.

$$
\sigma_{\text {nom }}^{v M}=\sqrt{3} \frac{16 T}{\pi D_{m}^{3}}
$$

The stress variation is inherently three dimensional in the shaft hub connection. The common assumption is however typically that for the strength evaluation we can assume the stress to be constant in the axial direction, although this is clearly not the case. In Figure 2 the result of a $3 \mathrm{D}$ contact modelling is shown. The hub is clamped at the outer surface and a linear varying distributed shear force is applied to the shaft end (linear in the radial direction with zero stress at the centre), symmetry condition is applied on the other shaft end and hub side (the surfaces not seen in Figure 2c). The result is a simulation of a clamped hub where a torsional load is applied to both shaft ends. We see a large axial variation in the stress (here von Mises) from the hub side to the hub centre (where the symmetry condition is applied).

The simulation time of this model with 1.8 million degrees of freedom is relatively long seen in relation to design optimization. The areas with the highest von Mises stress is where we have 


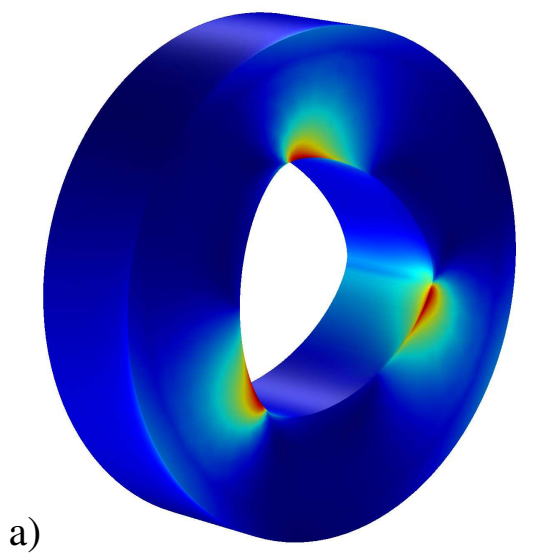

b)
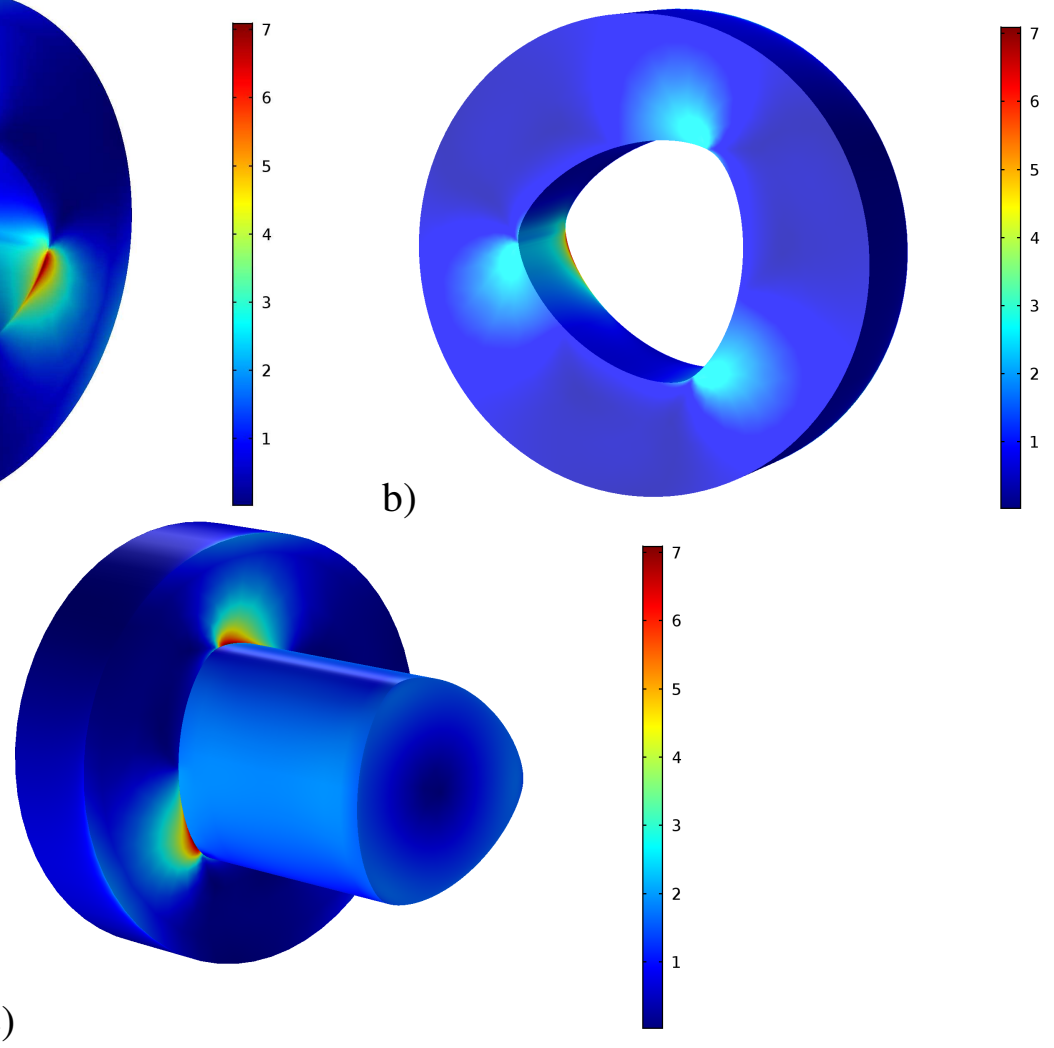

Figure 2: Illustration of variation in von Mises stress, the stress is scaled by the nominal von Mises stress. a) Free side of hub. b) Centre of hub (where symmetry condition is applied. c) Full model (half shaft and hub).

contact i.e. compressive stress. Looking instead at the principle stresses we see that the axial variation is primarily related to the compressive stress (smallest principle stress including sign) and less to the highest tensional stress (largest principle stress). In Figure 3 the principle stresses on the side of the hub is shown. The maximum compressive stress is numerically approximately twice as big as the largest tensional stress.

a)

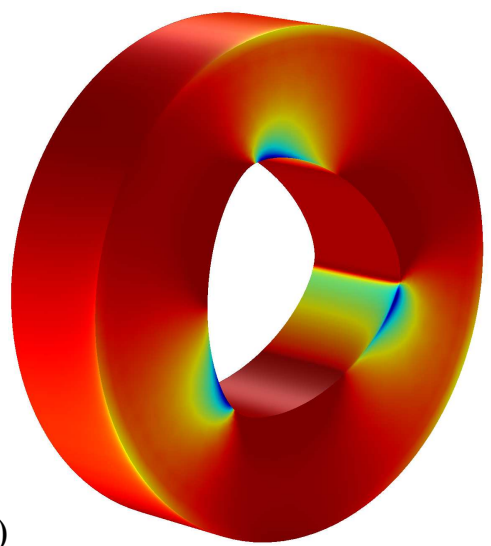

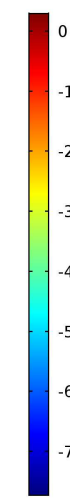

b)

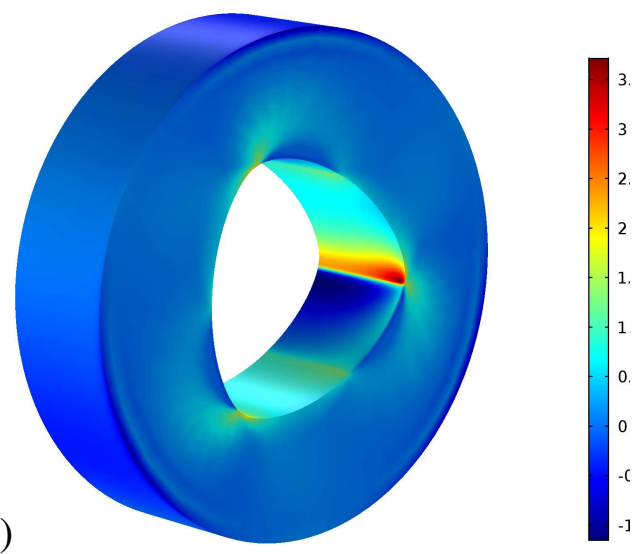

Figure 3: Illustration of variation in stress on the hub, the stress is scaled by the nominal von Mises stress. a) Largest compressive stress (smallest principle stress). b) Largest tensional stress (largest principle stress).

Both principle stresses are related to fatigue damage. The high compressive stress can be the reason for pitting damage as also seen in gear design. The largest tensile stress can lead to 
the initiation of cracks and final total hub failure, comparable to tooth breakage in gear design. Which of the two that is most critical will depend on the specific application. The pitting damage will lead to noise in the connection and loose of grip, but it will still be able to transfer power. For the case of failure due to the tensile stress the connection cannot transfer power.

From an optimization point-of-view, it can therefore be argued that a 2D FE model is sufficient if the objective is to reduce the maximum tensile stress. The introduction of torsion in a 2D model is however questionable, the boundary condition and the application of the load will have a high influence on the resulting stress distribution. We therefore in the present paper apply a full 3D analysis also for the optimization. To reduce the computational time we reduce the hub thickness to $1 / 10$ of the mean shaft diameter. This will result in higher stress value as compared to the natural choice of hub thickness equal to mean diameter of shaft (as seen in Figures 2 and 3).

The large variation in the stress in the axial direction for the 3D model is in fact a stress concentration (singularity) at the axial contact run out, as also discussed in (Pedersen 2016). With smaller and smaller elements towards this edge we will have an increase in the maximum stress. Although the stress is highest at the singularity we cannot use this for optimization purposes because we cannot guaranty the convergence. In relation to the contact, the modelling also needs to be highly refined. In Figure 4a the result of a second 3D contact modelling is shown, the hub thickness is reduced to $1 / 10$. The hub is also here clamped at the outer surface and a linearly varying shear force is applied to the shaft end, symmetry condition is applied on the other shaft end and hub side. Here we relative to Figure 2 have reduced the model to one third by applying cyclic symmetry conditions. The refined mesh is shown in Figures $4 \mathrm{~b}$ and $4 \mathrm{c}$, the model has 0.6 million degrees of freedom.

a)

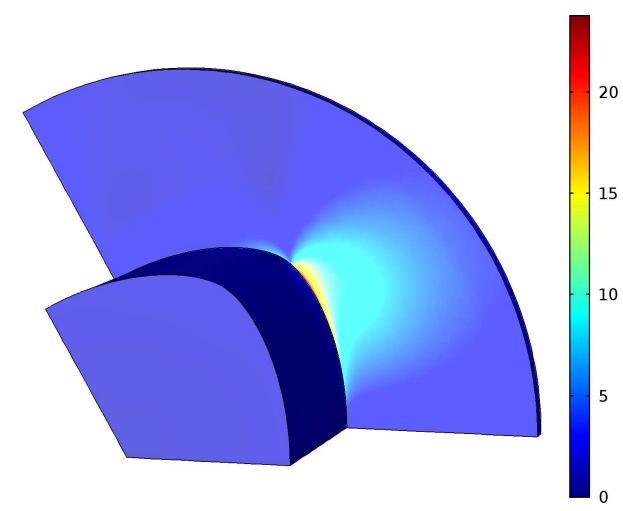

b)

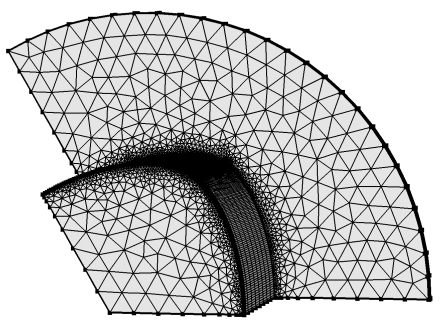

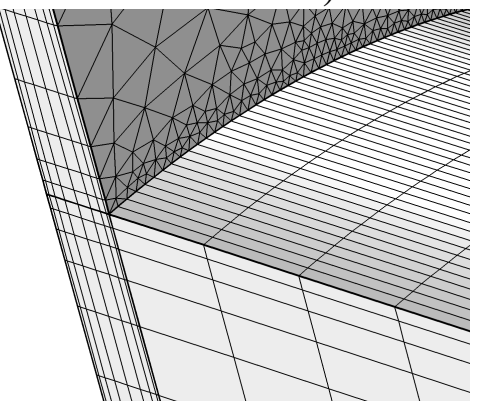

c)

Figure 4: Illustration of variation in von Mises stress, the stress is scaled by the nominal von Mises stress. a) Full model (1/6 of full shaft and hub model). b) FE mesh. c) Zoom of FE mesh.

In Figure 5 we show the variation in von Mises stress in the hub hole. The stress is shown along (counter clockwise) the outer rim of the part of the hole seen in Figure 4, and for the centre of the hole. Comparing the two curves we see a stress increase of about 2 times in the 
part where we have contact while the stress is more or less identical for the part of the hole not in contact with the shaft. The stress on the centre rim are converged by the selected mesh this is not to the same degree valid for the stress on the outer rim (that includes the singularity). From an optimization point-of-view it can be argued that we can perform the stress optimization on the centre curve since this will have a corresponding effect on the stress on the outer rim (verified by later examples).

The shown values of the stress concentration factor in Figure 5 are high but this is primarily related to the selected hub thickness.

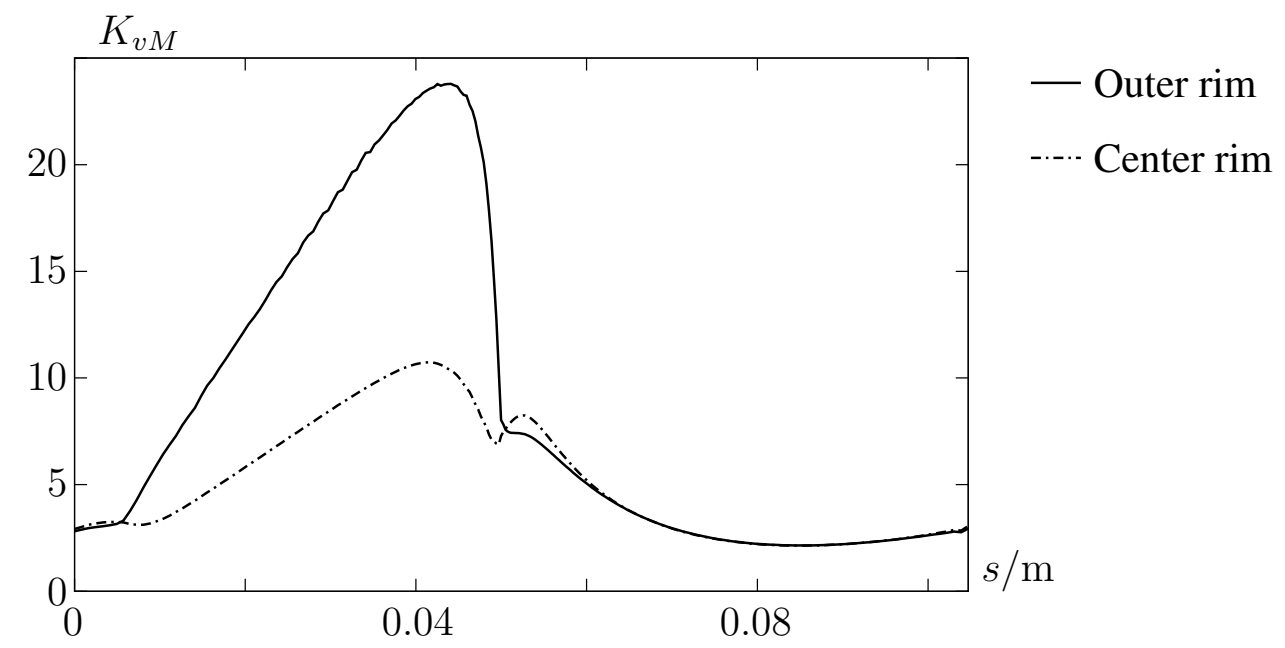

Figure 5: Variation in stress concentration factor. The stress is shown for the outer rim of the hole and the centre rim (lying in the symmetry plane) of the hole.

The result presented in Figure 2 is found with the assumption of a perfect fit between shaft and hub, i.e. no tolerance variation, and a zero friction coefficient. The influence from a smaller shaft than hub hole is investigated, the initial rigid body rotation is found from (21). The influence from tolerances (formulated as a smaller mean shaft diameter relative to the hub but still with the same eccentricity) is seen in Figure 6. The results are presented for four values of mean diameter for the shaft $D_{m}^{s}$, the mean hub diameter $D_{m}^{h}$ is kept constant.

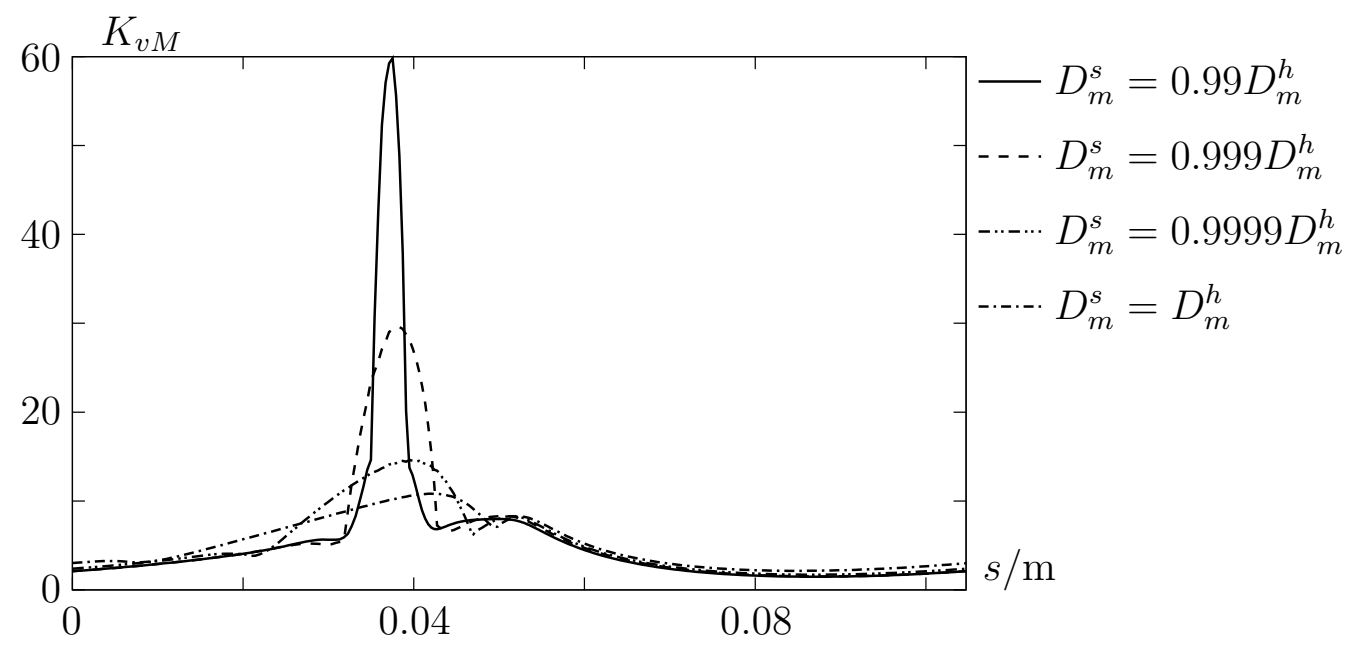

Figure 6: Variation in stress concentration factor for different values of tolerances. The stress is shown at the centre rim of the hole.

The results clearly show an increase in the stress for the contacting part as expected, the influence on the tensile part is however limited. 
The final investigation is in relation to the inclusion of friction or not. The friction is here modelled as Coulomb friction with a friction coefficient of $\mu=0.1$. In Figure 7 we compare the results with or without friction and with or without tolerances.

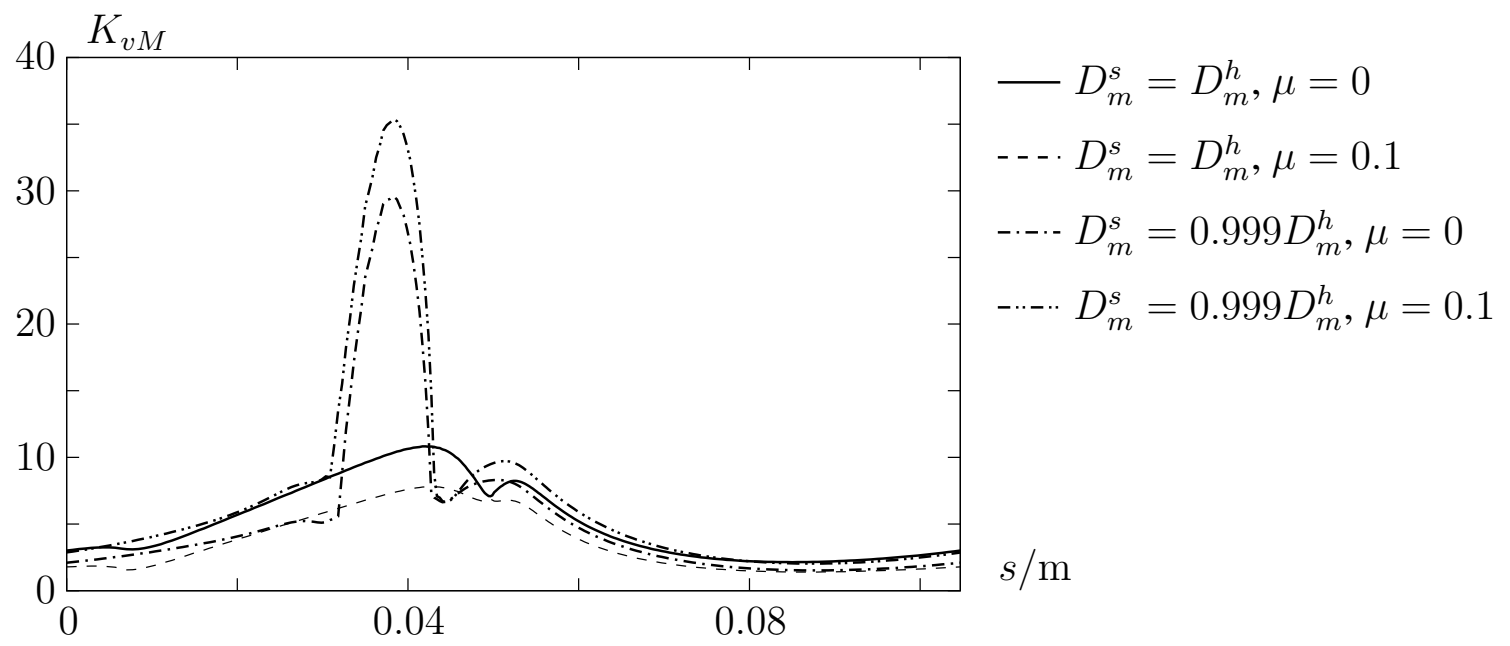

Figure 7: Variation in stress concentration factor for different values of tolerances. The stress is shown at the centre rim of the hole.

The results in Figure 7 clearly indicates that there is also an influence from including friction in the modelling. The difference between the curves with and without the inclusion of friction is primarily a scaling but also a change in the relationship between the maximum stresses on the contacting parts in tension.

The results in Figures 6 and 7 show that the stress level is affected by tolerances and the presence of friction. Due to the curve similarity and that the most generic case is the one with no tolerance, it is selected to perform the shape optimization of the positive connection without including tolerances. The influence from friction is included in the optimizations presented in the next section.

\section{Design optimization}

The results in the previous section are based on a specific choice of dimension and application of loads and boundary condition. The influence of the boundary condition is e.g. seen in Figure 2 where there is a stress increase at the clamped outer surface of the hub. Two factors control the size of a stress concentration, the shape at the point of maximum stress and the amount of load that is transferred to this point. For the specific selection made previously where the outer diameter of the hub equals two times the mean shaft diameter the stress variation along the rim of the hole will change if we swap the fixed boundary and the torsional load, i.e. if we fix the shaft end and apply a shear stress on the outer hub boundary. This is due to a change in the load path to the point of maximum stress. If we select a larger value for hub outer diameter this difference will decrease. If $D_{h o}=10 D_{m}$ we find that the stress variation is highly comparable relative to the swap of load and boundary condition, as seen in Figure 8.

The optimization is in the following therefore performed for two cases of hub diameter. Firstly, for a hub diameter defined by $D_{h o}=2 D_{m}$, which resembles more closely an actual situation, and secondly for the more generic case of $D_{h o}=10 D_{m}$ where the application of load and boundary condition can be exchanged. 


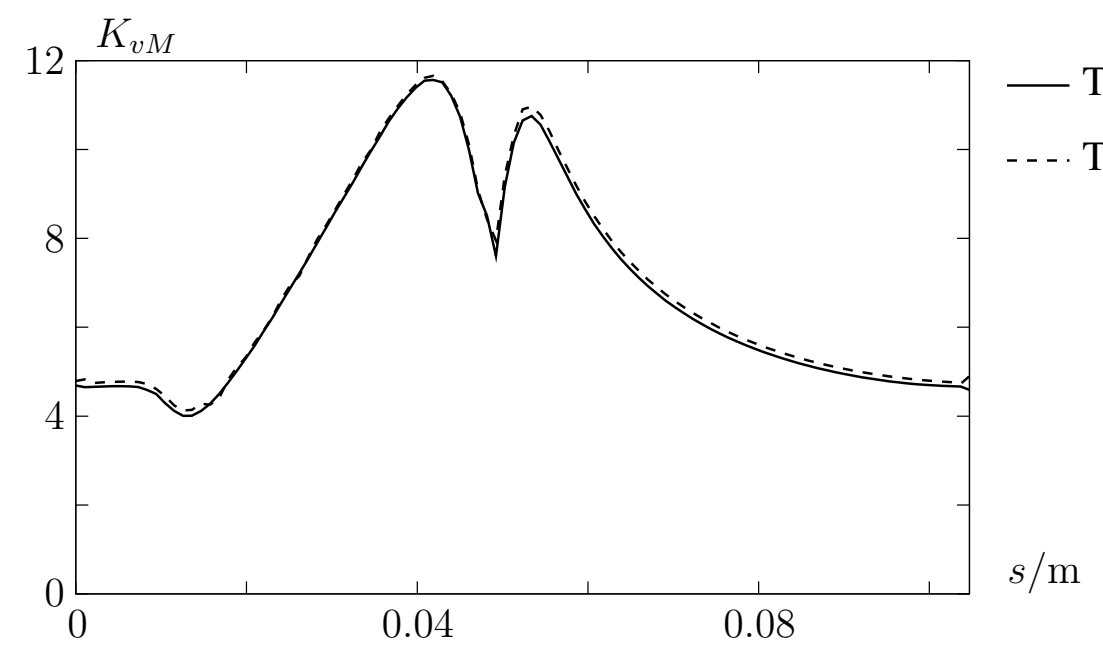

Figure 8: Variation in stress concentration factor for different application of load and boundary condition for the case $D_{h o}=10 D_{m}$ and $D_{m}=0.1 \mathrm{~m}$ for the specific design $e=0.045 D_{m}$. The stress is shown at the centre rim of the hole.

It is clear from the results of the previous section that the stress distribution is not optimal since the stress is not constant along a major part of the hole boundary. An obvious first choice is to find the optimal eccentricity value for a given mean diameter, i.e. keeping the trilobe shape design. The standard specifies the eccentricity for given mean diameter. For a diameter of $D_{m}=0.1 \mathrm{~m}$ the corresponding eccentricity is $e=0.045 D_{m}$, with these values, and using at first that $D_{h o}=2 D_{m}$, we get on the centre rim of the hole a stress concentration factor of $K_{v M}=10.8$ assuming that there is a perfect fit and no friction in the contact. The stress variation is shown in Figure 9. The stress variation is not optimal, the maximum stress is found in the contact not in the corner of the trilobe. To reduce the maximum stress in the contact we must increase the eccentricity because this will increase the pressure angle. Increasing the eccentricity will make the corner in the trilobe design smaller whereby the stress concentration found here is increased. The optimal design is therefore the value of eccentricity that balances these two points of maximum stress such that they are identical. In Figure 9 we also show the stress variation for a higher eccentricity value and for the best (for this case) eccentricity value, i.e. $e=0.0565 D_{m}$. The stress has for the best design been reduced $17 \%$ relative to the design specified by DIN 32711.

If we take friction into consideration, here with $\mu=0.1$ (corresponding to lubricated steelsteel contact), the curves are slightly shifted and the best design is found for $e=0.052 D_{m}$. Using the more generic case of $D_{h o}=10 D_{m}$ we find different optimal designs. The results are summarized in Table 1. In the table we also present the improvement in the stress level relative to the design with $e=0.045 D_{m}$ but with the same coefficient of friction.

We notice from the results in Table 1 that overall the stress level is decreased by including friction in the model. In (Ziaei \& Leidich 2003) the reported optimal eccentricity value is $e=$ $0.036 D_{m}$ with an assumed friction coefficient of $\mu=0.2$ this does not agree with the present paper findings. It should be noted that for $\mu=0.2$ the variation in maximum stress for different values of eccentricity is relatively flat around the optimal value. The reason that we in the present paper finds a different value of optimal eccentricity relative to (Ziaei \& Leidich 2003) might be due to the more refined FE modelling used here. It is notable that the DIN specified eccentricity seems to be the optimal value for larger values of the hub outer diameter when friction is included.

To improve the strength further a different hole parameterization is suggested. The hole 


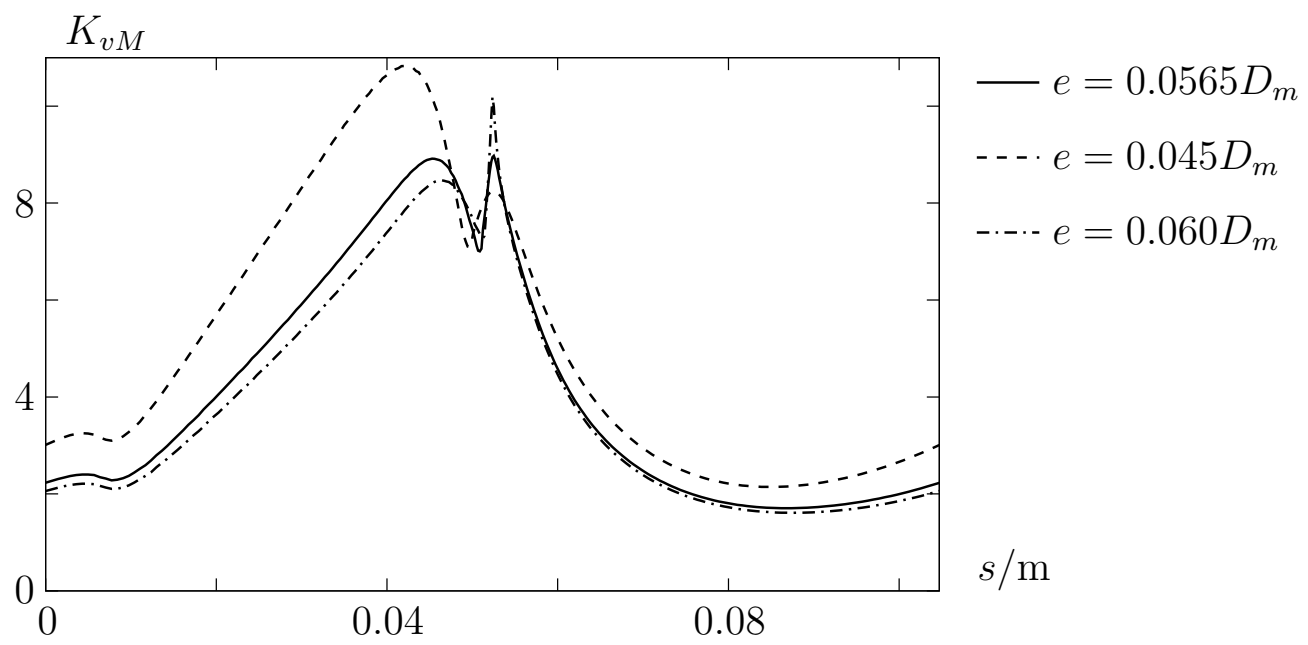

Figure 9: Variation in stress concentration factor for different values of eccentricity. The stress is shown at the centre rim of the hole.

\begin{tabular}{|c|c|c|c|c|}
\hline \multirow{2}{*}{$D_{h o} / D_{m}$} & \multicolumn{4}{|c|}{ Trilobe design } \\
\cline { 2 - 5 } & $\mu$ & $e / D_{m}$ & $K_{v M}$ & $\left(K_{v M}^{\mathrm{DIN}}-K_{v M}\right) / K_{v M}^{\mathrm{DIN}}$ \\
\hline \hline 2 & 0.0 & 0.00565 & 8.93 & 0.17 \\
2 & 0.1 & 0.0052 & 7.26 & 0.07 \\
2 & 0.2 & 0.0046 & 6.80 & 0.01 \\
\hline \hline 10 & 0.0 & 0.0050 & 10.8 & 0.08 \\
10 & 0.1 & 0.0045 & 8.62 & 0.0 \\
10 & 0.2 & 0.0045 & 7.56 & 0.0 \\
\hline
\end{tabular}

Table 1: Optimized values of design parameters for trilobe design. The new improved stress concentration factors are also shown, and the improvement relative to the DIN specified design.

shape can be parameterized in an infinite number of ways, the focus in the present paper is to limit the number of design parameters. From the results presented in Figure 9 it is clear that the design presented is not optimal with respect to minimum stress, the design was/is primarily selected because of the possibility of production by grinding. With today's production possibilities we can use more appropriate shapes that can lower the stress concentration even further. In machine elements it is always a balance between the benefits of a specific design and the practical design realization.

In the present paper we select to use a variation of the super elliptical shape. In general the super ellipse is in parametric form given as

$$
\begin{array}{ll}
X=a \cos (t)^{(2 / \eta)}, & t \in\left[0: \frac{\pi}{2}\right] \\
Y=b \sin (t)^{(2 / \eta)}, & t \in\left[0: \frac{\pi}{2}\right]
\end{array}
$$

for one quarter, here $\eta$ is the super elliptic power and $a$ and $b$ are the principal axes. We choose here to stick to a triangular design domain, which puts some constraints on the design. In order to compare the design to the trilobe we select that the new design lies within a circle with a diameter equal to the maximum diameter for the trilobe which is $D_{\max }=D_{m}+2 e_{\max }=$ $9 D_{m} / 8$.

The design space is the triangle shown in Figure 10.

The idea is to connect point $A$ and $B$ by the super ellipse, and then copy this shape between 


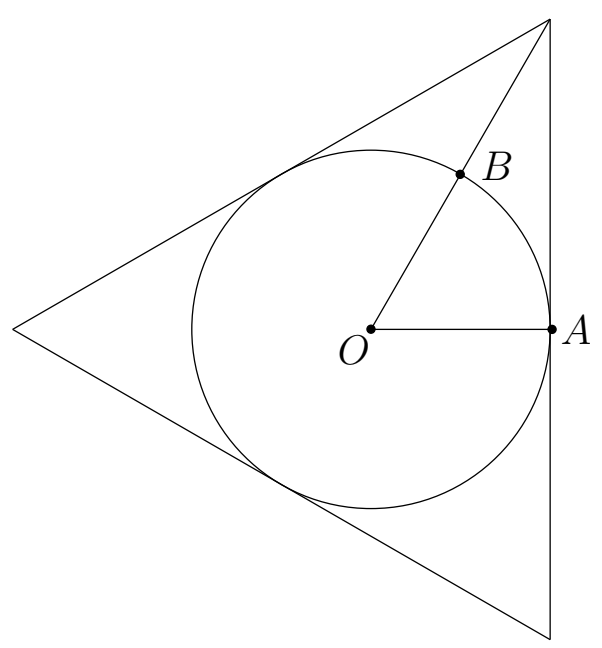

Figure 10: Design domain.

points $A$ and $B$ six times to have the full new shaft design (by first mirror the shape around the line from $O$ to $B$ and then copy this design by rotating two times). The design variables thereby includes $L_{1}=|O A|$, i.e. the distance from point $O$ to $A$ and $L_{2}=|O B|$, i.e. the distance from point $O$ to $B$. According to the previous choice we therefore fix the distance $L_{2}$ to be the maximum value, i.e. $L_{2}=9 D_{m} / 16=0.05625 \mathrm{~m}$ under the assumption that $L_{1}<L_{2}$. In order to have continuity in the slope of the shaft shape the super ellipse is distorted as also described in (Pedersen 2015). The distorted super ellipse is in parametric form given as

$$
\begin{aligned}
& X=a \cos (t)^{(2 / \eta)}, t \in\left[0: \frac{\pi}{2}\right] \\
& Y=\left(b-a \cos (t)^{(2 / \eta)} \tan (\gamma)\right) \sin (t)^{(2 / \eta)}, t \in\left[0: \frac{\pi}{2}\right]
\end{aligned}
$$

The super ellipse and the distorted super ellipse are illustrated in Figure 11, where also the angle $\gamma$ is defined.
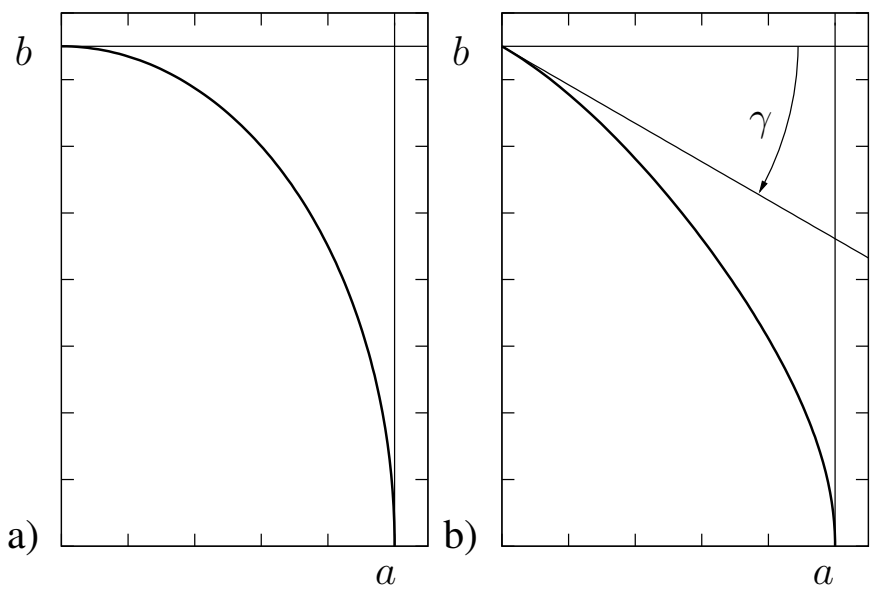

Figure 11: a) Super ellipse. b) Distorted super ellipse.

For the selected design the distortion angle is fixed at $\gamma=\pi / 6$, the principal axes are $a=$ $L_{1}-L_{2} / 2$ and $b=\sqrt{3} L_{2} / 2$. This leaves only two free design parameters, $\phi=L_{2} / L_{1}$ and $\eta$. With the given simple parameterization we can fill the whole triangular design domain in figure 10 with a shape that has no discontinuity in the slope (points of infinite stress in a FE model). 
Assuming no friction in the contact the optimization is first performed for $D_{h o}=10 D_{m}$, with $D_{m}=0.1 \mathrm{~m}$, relating it to the previous result for the trilobe shape. The optimal values are $\phi=1.49$ and $\eta=3.40$, the maximum stress on the centre rim of the hole is reduced to $K_{v M}=6.66$. This corresponds to a reduction in the maximum stress of $44 \%$ relative to the DIN specified design.

The same optimizations as presented in Table 1 are performed with the super elliptical shape, the results are summarized in Table 2. Overall, the results show that large improvements in the stress can be found when comparing to the trilobe design.

\begin{tabular}{|c|c|c|c|c|c|}
\hline & \multicolumn{5}{|c|}{ Distorted super elliptical design } \\
\cline { 2 - 6 }$D_{h o} / D_{m}$ & $\mu$ & $\phi$ & $\eta$ & $K_{v M}$ & $\left(K_{v M}^{\mathrm{DIN}}-K_{v M}\right) / K_{v M}^{\mathrm{DIN}}$ \\
\hline \hline 2 & 0.0 & 1.56 & 3.35 & 5.81 & 0.46 \\
2 & 0.1 & 1.47 & 3.50 & 5.36 & 0.31 \\
2 & 0.2 & 1.31 & 3.70 & 5.07 & 0.26 \\
\hline \hline 10 & 0.0 & 1.50 & 3.40 & 6.62 & 0.44 \\
10 & 0.1 & 1.42 & 3.55 & 6.01 & 0.30 \\
10 & 0.2 & 1.32 & 3.50 & 5.84 & 0.27 \\
\hline
\end{tabular}

Table 2: Optimized values of design parameters for distorted super elliptical design. The new improved stress concentration factors are also shown, and the improvement relative to the DIN specified design (DIN 32711).

The optimized design is seen from the table to be a function of both outer diameter and the friction coefficient. The objective is most sensitive to the selected $\phi$ and less to the super elliptical power $\eta$.

The specific stress concentration factor presented in Tables 1 and 2 is to a large extent depending on the selected hub thickness, as discussed previously. The FE mesh discretization also has a minor influence, but the simulations are all made with similar refinements so that the relative improvements as presented are valid.

The most generic selection is probably the design for $\mu=0.1$ and the larger outer diameter. The stress variation for this design is compared to the design specified by DIN 32711 in Figure 12. We notice that more than one point on the stress curve reach the maximum level for the optimized super elliptical design. With a more involved shape parameterization it can most probably be reduced further, but the focus here is on a simplified design with few design parameters.

The optimized design shape and the stress level in the hub for the case of $D_{h o}=10 D_{m}$, and $\mu=0.1$ is shown in Figure 13, please notice the reduction of the stress on the outer rim as compared to the design in Figure 4, although the optimization has been performed for the stress on the centre rim of the hub.

The general conclusion is that a generic optimized design could be selected having the design values $\phi=1.4$ and $\eta=3.5$. In Figure 14 the super elliptical design is compared to the trilobe design with the eccentricity as specified by DIN 37211. The improvement of the new designs relative to the fatigue strength is directly related to the reduction in the stress concentration.

\section{Conclusion}

Torque transmission between shaft and hub can be designed in many ways and the designs are given in different standards. In the present paper the focus is on geometrically locked connections and the starting point is polygon connection specified by DIN 32711 (P3G). In 


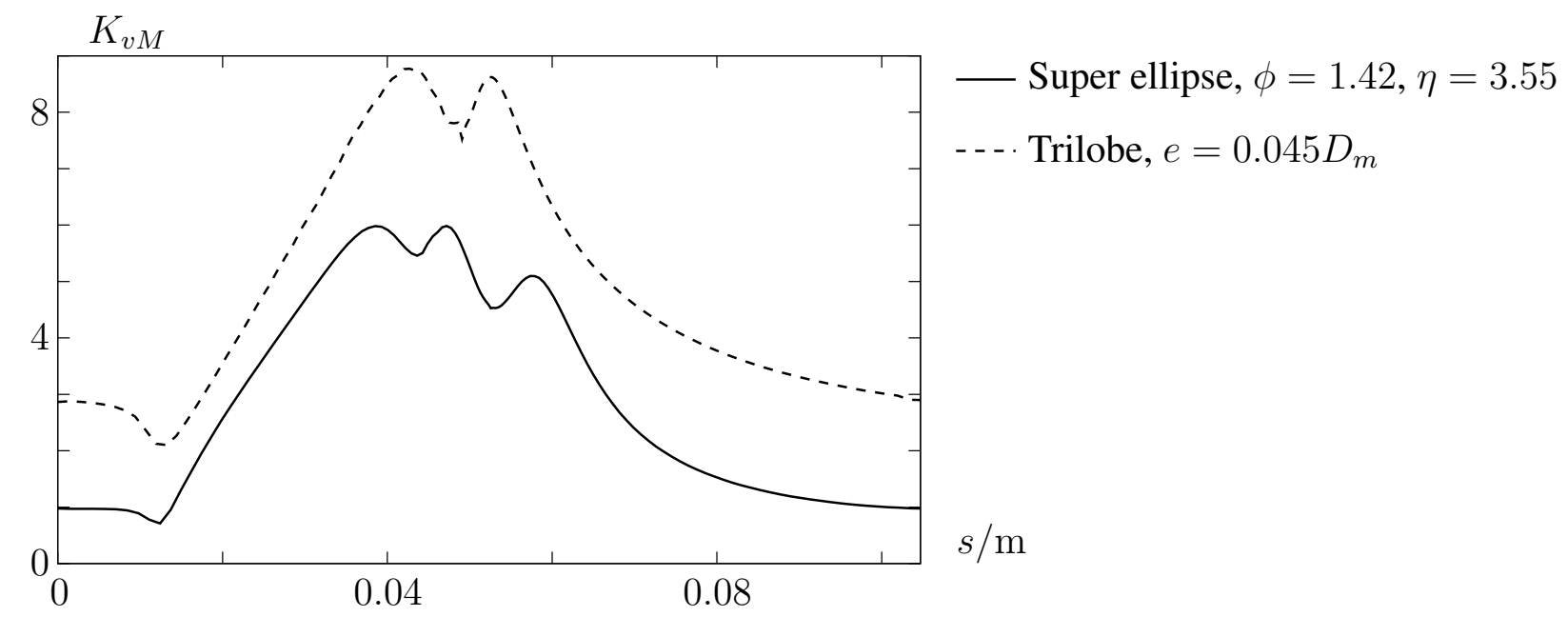

Figure 12: Variation in stress concentration factor for optimized design and design specified by DIN 32711 for $D_{h o}=10 D_{m}$, and $\mu=0.1$. The stress is shown at the centre rim of the hole.

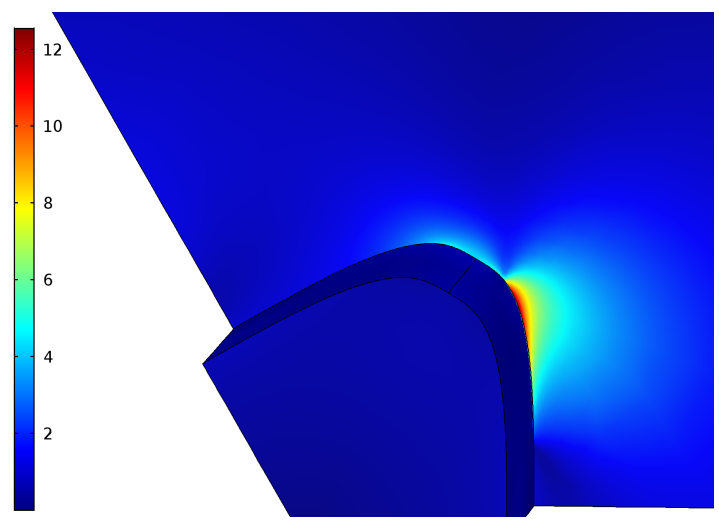

Figure 13: Illustration of variation in von Mises stress for optimized super elliptical design with $D_{h o}=10 D_{m}$ and $\mu=0.1$, the stress is scaled by the nominal von Mises stress.

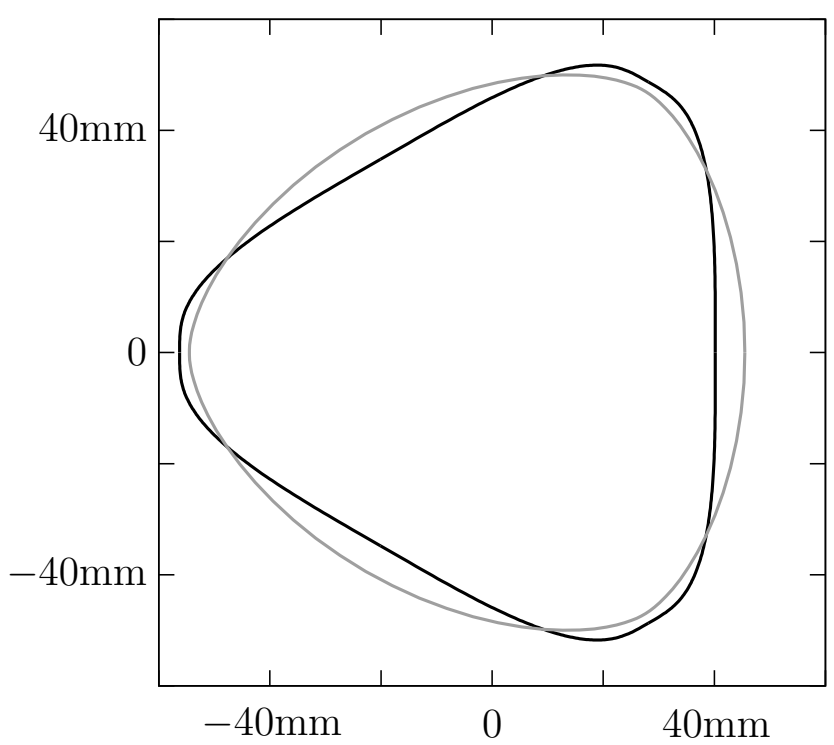

- Super ellipse, $\phi=1.4, \eta=3.5$

Trilobe, $e=0.045 D_{m}$

Figure 14: Comparison of suggested super elliptical design and trilobe design as specified by DIN 37211.

order to evaluate a design improvement we have to be able to analyse the stress level in the 
connection sufficiently correct. For a geometrically locked connection we must include contact analysis and at the same time the torque must be applied to either the shaft or the hub while the counter part is fixed. For this reason it is in the present paper chosen to use a full 3D finite element analysis. Analysis and design of shaft-hub polygon connections is a challenging 3D problem with a number of influencing parameters that are extensively discussed.

The value of a theoretical stress concentration depends on two factors; the load size that is transferred to the place of interest and the specific shape at this point. The stress variation found from an analysis therefore depends on the outer hub diameter size and the shaft length, since this is where the torque or the boundary condition are applied. We select here to have these dimensions so large that the influence from swapping the torque and the boundary condition is limited. In the paper it is also examined how large an influence we have on the stress level from including tolerance and the friction. Both are shown to have an influence on the stress level. The tolerances are neglected for the evaluation of optimized designs whereas the influence of friction is examined.

A new triangular shape design is presented, the design is based on the simple super ellipse. The design optimization shows that large strength improvements in the stress level can be found, typically in the order of $20 \%$ to $40 \%$ depending on the specific friction coefficient value. The improvements are given relative to the trilobe design as specified in DIN 32711, and under the assumption that the new design has a largest diameter equal to the diameter specified by the standard, i.e. the new design can lie within the trilobe design.

\section{Acknowledgment}

For discussions and suggestions I wish to thank Prof. Peder Klit and Prof. Pauli Pedersen.

\section{References}

Bhatta, R. \& Reffeor, W. (2017), 'Comparison of load carrying capacity of three and four lobed polygonal shaft and hub connection for constant grinding diameter', Proceedings of the Asme International Mechanical Engineering Congress and Exposition, 2016, Vol. 9.

Citarella, R. \& Perrella, M. (2015), 'Robust design of a polygonal shaft-hub coupling', Frattura ed Integrita Strutturale 9, 554-563.

COMSOL AB (1986 -), Stockholm, Sweden, www.comsol.com.

Czyzewski, T. \& Odman, M. T. (1988), 'Analysis of contact stress and deformation in a trilobe polygonal connection', Journal of Engineering for Industry 110(3), 212.

DIN 32711 (1979), 'Polygonprofile P3G; antriebselemente (in German)'.

DIN 32712 (1979), 'Polygonprofile P4C; antriebselemente (in German)'.

Ding, Y. (1986), 'Shape optimization of structures: A literature survey', Computers and Structures 24(6), 985-1004.

Fessler, H. \& Moore, C. J. (1996), 'Stresses in a splineless drive for aero-engines', Proceedings of the Institution of Mechanical Engineers, Part G: Journal of Aerospace Engineering 210(2), 189-202.

Filemon, E. (1959), 'Production and analysis of polygon profiles', Periodica polytechnica M III/1. 
Großmann, C. (2007), Fretting fatigue of shape optimised polygon-shaft-hub connections, Berlin University.

Großmann, C. \& Ziaei, M. (2004), 'Optimierung der nach DIN 32712 genormten p4cpolygon-welle-nabe- verbindungen, optimisation of standardised p4c-polygon-shaft-hubconnections', Konstruktion (11-12), 70-74.

Kahn-Jetter, Z. L., Hundertmark, E. \& Wright, S. (2000), 'Comparison of torque transmitting shaft connectivity using a trilobe polygon connection and an involute spline', Journal of Mechanical Design, Transactions of the Asme 122(1), 130-135.

Pedersen, N. L. (2010), 'Stress concentrations in keyways and optimization of keyway design', Journal of Strain Analysis for Engineering Design 45(8), 593-604.

Pedersen, N. L. (2011), 'Optimization of straight-sided spline design', Archive of Applied Mechanics 81(10), 1393-1407.

Pedersen, N. L. (2013), 'Overall bolt stress optimization', Journal of Strain Analysis for Engineering Design 48(3), 155-165.

Pedersen, N. L. (2015), 'Minimizing tooth bending stress in spur gears with simplified shapes of fillet and tool shape determination', Engineering optimization 47(6), 805-824.

Pedersen, N. L. (2016), 'On optimization of interference fit assembly', Structural and Multidisciplinary Optimization 54(2), 349-359.

Pedersen, N. L. (2019), 'Stress concentration and optimal design of pinned connections', Journal of Strain Analysis for Engineering Design 54(2), 95-104.

Ziaei, M. (2010), 'Calculation of the torsional- and contact stresses in the new nonvolatile $\mathrm{m}$-profiles and comparison with the standard involute tooth profiles according to DIN 5480', VDI Berichte: Welle-nabe-verbindungen: Gestaltung - Fertigung - Anwendungen Mit Fachausstellung 2114, 231-246.

Ziaei, M. \& Leidich, E. (2003), 'Optimierung des tragverhaitens von genormten polygonprofilen nach DIN 32711 und DIN 32712', Vdi Berichte (1790), 91-108. 